\title{
Article \\ Effect of Genetic Variability in 20 Pharmacogenes on Concentrations of Tamoxifen and Its Metabolites
}

\author{
Yuanhuang Chen ${ }^{1} \mathbb{D}$, Lauren A. Marcath ${ }^{2} \mathbb{D}$, Finn Magnus Eliassen ${ }^{3}$, Tone Hoel Lende ${ }^{3}$, Havard Soiland ${ }^{4,5}$, \\ Gunnar Mellgren ${ }^{4,5}$, Thomas Helland ${ }^{1,4,5}$ and Daniel Louis Hertz ${ }^{1, * \mathbb{D}}$
}

1 Department of Clinical Pharmacy, University of Michigan College of Pharmacy, Ann Arbor, MI 48109-1065, USA; cyuanh@umich.edu (Y.C.); hellandt@med.umich.edu (T.H.)

2 Department of Pharmacotherapy, Washington State University College of Pharmacy \& Pharmaceutical Sciences, Spokane, WA 99202, USA; lauren.marcath@wsu.edu

3 Department of Breast and Endocrine Surgery, Stavanger University Hospital, P.O. Box 8100, 4068 Stavanger, Norway; finnmagnus@gmail.com (F.M.E.); tone.hoel.lende@sus.no (T.H.L.)

4 Department of Clinical Science, University of Bergen, 5021 Bergen, Norway; hsoiland@gmail.com (H.S.); Gunnar.Mellgren@uib.no (G.M.)

5 Hormone Laboratory, Department of Medical Biochemistry and Pharmacology, Haukeland University Hospital, 5021 Bergen, Norway

* Correspondence: DLHertz@med.umich.edu; Tel.: +1-734-763-0015; Fax: +1-734-763-4480

check for updates

Citation: Chen, Y.; Marcath, L.A.; Eliassen, F.M.; Lende, T.H.; Soiland, H.; Mellgren, G.; Helland, T.; Hertz, D.L. Effect of Genetic Variability in 20 Pharmacogenes on Concentrations of Tamoxifen and Its Metabolites. J. Pers Med. 2021, 11, 507. https://doi.org/ 10.3390/jpm11060507

Academic Editors: Werner Schroth and Matthias Schwab

Received: 4 May 2021

Accepted: 31 May 2021

Published: 4 June 2021

Publisher's Note: MDPI stays neutral with regard to jurisdictional claims in published maps and institutional affiliations.

Copyright: (c) 2021 by the authors. Licensee MDPI, Basel, Switzerland. This article is an open access article distributed under the terms and conditions of the Creative Commons Attribution (CC BY) license (https:/ / creativecommons.org/licenses/by/ $4.0 /)$.

\begin{abstract}
Background: Tamoxifen, as a treatment of estrogen receptor positive (ER+) breast cancer, is a weak anti-estrogen that requires metabolic activation to form metabolites with higher anti-estrogenic activity. Endoxifen is the most-studied active tamoxifen metabolite, and endoxifen concentrations are highly associated with CYP2D6 activity. Associations of tamoxifen efficacy with measured or CYP2D6-predicted endoxifen concentrations have been inconclusive. Another active metabolite, 4-OHtam, and other, less active metabolites, Z-4'-endoxifen and Z-4'-OHtam, have also been reported to be associated with tamoxifen efficacy. Method: Genotype for 20 pharmacogenes was determined by VeriDose ${ }^{\circledR}$ Core Panel and VeriDose ${ }^{\circledR}$ CYP2D6 CNV Panel, followed by translation to metabolic activity phenotype following standard activity scoring. Concentrations of tamoxifen and seven metabolites were measured by UPLC-MS/MS in serum samples collected from patients receiving $20 \mathrm{mg}$ tamoxifen per day. Metabolic activity was tested for association with tamoxifen and its metabolites using linear regression with adjustment for upstream metabolites to identify genes associated with each step in the tamoxifen metabolism pathway. Results: A total of 187 patients with genetic and tamoxifen concentration data were included in the analysis. CYP2D6 was the primary gene associated with the tamoxifen metabolism pathway, especially the conversion of tamoxifen to endoxifen. CYP3A4 and CYP2C9 were also responsible for the metabolism of tamoxifen. CYP2C9 especially impacted the hydroxylation to 4-OHtam, and this involved the OATP1B1 (SLCO1B1) transporter. Conclusion: Multiple genes are involved in tamoxifen metabolism and multi-gene panels could be useful to predict active metabolite concentrations and guide tamoxifen dosing.
\end{abstract}

Keywords: pharmacogenetics; tamoxifen; endoxifen; active metabolites; CYP2D6; CYP3A4; CYP2C9; SLCO1B1; breast cancer

\section{Introduction}

Tamoxifen is a selective estrogen receptor modulator used in the adjuvant treatment of hormone receptor positive (HR+), i.e., estrogen receptor and/or progesterone receptor positive breast cancer. Tamoxifen use for 5-10 years significantly reduces the risk of recurrence and improves survival among patients with HR+ breast cancer; however, the 10-year recurrence rate is up to $30 \%$ [1]. Tamoxifen is a weak anti-estrogen that requires metabolic activation. The major route of tamoxifen metabolic activation occurs through the demethylation of tamoxifen to N-desmethyl-tamoxifen (NDtam) followed by 4-hydroxylation of NDtam to the active metabolite 4-hydroxy-N-desmethyl-tamoxifen (endoxifen). A second 
active metabolite, 4-hydroxy-tamoxifen (4OHtam), is formed by 4-hydroxylation of tamoxifen. Endoxifen and $4 \mathrm{OH}$ tam have $\sim 100 \times$ higher affinity to the estrogen receptor compared to the parent drug tamoxifen [2]. Previous pre-clinical and pharmacological studies indicate that these two active metabolites may be responsible for the anti-estrogenic effect and efficacy of tamoxifen [3-6]. Although several previous studies found an association between endoxifen systemic concentrations and tamoxifen treatment outcomes, two studies also reported that low $4 \mathrm{OH}$ tam levels were associated with worse treatment outcome [3,4]. There are additional tamoxifen metabolites that may be partially responsible for tamoxifen's anti-estrogenic activity, such as $4^{\prime}$-hydroxy-N-desmethyl-tamoxifen (Z-4'-endoxifen) and $4^{\prime}$-hydroxy-tamoxifen (Z-4'-OHtam). These 4-prime $\left(4^{\prime}\right)$ metabolites were reported to have $\sim 10 \%$ of the anti-estrogenic activity of their Z-isomer counterparts [7]. However, detection and quantification of the $4^{\prime}$-metabolites requires chromatographic separation, as their molecular masses and fragmentation patterns are similar to other isomeric forms of endoxifen and $4 \mathrm{OHtam}$. Several studies have used assays that did not account for this and, therefore, reported over-estimated concentrations of un-separated isoforms of endoxifen and $4 \mathrm{OH}$ tam [8]. Therefore, algorithms that include endoxifen and other active metabolites may be helpful to confirm the association of tamoxifen metabolite concentrations with tamoxifen treatment efficacy [9].

Endoxifen levels are highly variable among patients receiving $20 \mathrm{mg} /$ day tamoxifen, and a major source of endoxifen variability is attributed to the activity of $C Y P 2 D 6$, the ratelimiting enzyme in the conversion of NDtam to endoxifen. The gene encoding CYP2D6 is highly polymorphic, with over 100 allelic variants/haplotypes known, including several common variants associated with reduced or abolished CYP2D6 phenotypic activity [10]. The CYP2D6 genotype explains up to $50 \%$ of endoxifen variability and around $9 \%$ of $4 \mathrm{OHtam}$ variability [11]; thus, there are additional factors contributing to the formation of these two active metabolites [12]. Tamoxifen metabolism is complex and involves several other genes that may be predictive of endoxifen and $4 \mathrm{OH}$ tam concentrations, including CYP2Cs, CYP3As, SULTs, and UGTs [12]. The generation of active metabolites may require multiple metabolic steps for which genes responsible for upstream metabolism may affect concentrations of the downstream active metabolite. For example, endoxifen formation requires two steps: first, tamoxifen is demethylated to NDtam by one of several enzymes, and then it is hydroxylated to endoxifen, which is catalyzed by CYP2D6 alone. Genes responsible for the generation of NDtam may, therefore, also be predictive of endoxifen formation. The same is true for Z $\mathrm{Z}-4^{\prime}$-endoxifen and nor-endoxifen, which are secondary and tertiary metabolites of tamoxifen, respectively. It was previously reported that CYP2B6 and CYP2D6 contribute to the formation of Z-4'-OHtam, but the genes associated with $Z-4^{\prime}$-endoxifen remain unknown [11]. Few in-vivo studies on breast cancer patients using tamoxifen have investigated the genetic variables that affect non-endoxifen metabolites, as was recently reviewed by our group [12]. The objective of this study was to elucidate the effect of variation in 20 pharmacogenes on concentrations of tamoxifen and seven of its metabolites, measured by a gold-standard LCMSMS methodology.

\section{Materials and Methods}

\subsection{Patient Cohort}

Patients with breast cancer using adjuvant tamoxifen were recruited through the Prospective Breast Cancer Biobank, a prospective population-based biobank project collecting liquid biopsies and clinical data from breast cancer patients recruited at Haukeland and Stavanger University Hospitals in Norway [13]. Patients with estrogen receptor positive $(\mathrm{ER}+)$ breast cancer who were recommended tamoxifen as primary endocrine adjuvant treatment $(n=220)$ were included in a previous analysis investigating the associations of tamoxifen metabolite concentrations with treatment-related side effects [14]. From this population, patients with stored whole-blood samples for germline DNA purification were included in the current study. Data on CYP2D6 inhibitor-use were obtained through the Norwegian Prescription database. All participants provided written informed consent 
before enrolling to the Prospective Breast Cancer Biobank and the biobank is approved by the Norwegian Regional Ethical Committee (2010/1957 and 2011/2161).

\subsection{Sample Collection and Quantification of Tamoxifen and Metabolites}

Serum samples drawn at least one month after tamoxifen initiation were collected in Vacuette $^{\mathrm{TM}}$ serum tubes containing clot activator, coagulated for 30-60 min, centrifuged for $10 \mathrm{~min}$ at $2200 \mathrm{~g}$ and stored at $-80^{\circ} \mathrm{C}$. EDTA whole-blood samples were collected on the day of surgery and stored at $-80^{\circ} \mathrm{C}$. All patients were given instructions not to take anti-hormonal drugs on the morning of the blood draw.

Steady-state concentrations of tamoxifen, N-desmethyl-tamoxifen (NDtam), Z-4hydroxy-tamoxifen (Z-4OHtam), $4^{\prime}$ isomer of Z-4OHtam (Z-4'-OHtam), Z-4-hydroxy-Ndesmethyl-tamoxifen (Z-endoxifen), $4^{\prime}$ isomer of Z-endoxifen (Z-4'-endoxifen), tamoxifen$\mathrm{N}$-oxide (TamNoX), and N-N-didesmethyl-tamoxifen (NNDDtam) were assessed using a validated UPLC-MS/MS method for serum [14]. All metabolites and four deuterated internal standards (Tamoxifen-d5, 4OHNDtam-d5, Z-4OHtam-d5, NDtam-d5) were obtained commercially. Briefly, $20 \mu \mathrm{L}$ serum samples were pre-processed using a Hamilton STAR pipetting robot (Bonaduz, Switzerland) and the resulting $80 \mu \mathrm{L}$ supernatant was evaporated to dryness using nitrogen and reconstituted in $500 \mu \mathrm{L}$ water:methanol (20:80, $\mathrm{v}: \mathrm{v})$. The samples were chromatographically separated by an Aquity UPLC system from Waters (Milford, MA, USA) using a Waters BEH Phenyl column before being subjected to atmospheric pressure photoionization and detected in positive ion mode using a Xevo TQ-S tandem mass spectrometer (Waters).

\subsection{Germline DNA Isolation, Genotyping, and Activity Phenotype Prediction}

Germline DNA was extracted from $400 \mathrm{uL}$ whole blood at the ISO certified (ISO9001) HUNT biobank, NTNU, Levanger, Norway, using the fully automated Chemagic Star workstation for nucleic acid extraction, Hamilton (Bonaduz, Switzerland). DNA was genotyped on the VeriDose ${ }^{\circledR}$ Core Panel and VeriDose ${ }^{\circledR}$ CYP2D 6 CNV Panel at Agena Bioscience (San Diego, CA, USA). The VeriDose ${ }^{\circledR}$ Core Panel tested 68 SNPs/INDELs in twenty genes and five CNV assays. This panel was selected to provide broad coverage of the major functionally consequential genetic variability in important pharmacogenes, including polymorphic enzymes and transporters. The VeriDose ${ }^{\circledR}$ CYP2D6 CNV Panel includes seven regions in the CYP2D6 gene and $22 \mathrm{CNV}$ assays. This panel was selected to ensure that we had comprehensive genomic coverage of CYP2D6, which is known to be the most important pharmacogene in tamoxifen metabolism. All genetic information underwent appropriate quality control, including the assessment of sample call rate. Similar to our previous study [15], each allele was translated into the predicted activity ( $\mathrm{N}=\mathrm{Normal}$, $\mathrm{L}=$ Low, $\mathrm{H}=\mathrm{High}$ ) based on published data and CPIC guidelines when available (Supplementary Table S1). Each patient's diplotype was translated into predicted activity phenotypes $(\mathrm{PM}=$ Poor Metabolizer, $\mathrm{IM}=$ Intermediate Metabolizer, $\mathrm{NM}=$ Normal Metabolizer, RM = Rapid Metabolizer, UM = Ultra-rapid Metabolizer) for analysis (Supplementary Table S2), including CYP2D6, which was translated using consensus activity score designations from current CPIC guidelines [16].

\subsection{Statistical Analysis and Data Analyses}

Patients who had metabolite concentrations available and underwent successful genotyping were included in the analysis. Patients receiving a dose other than $20 \mathrm{mg} /$ day $(n=1)$ or those with steady-state tamoxifen concentration $<40 \mathrm{nM}(n=15)$, who were considered to be non-adherent to tamoxifen [5], were excluded from analyses. The data of tamoxifen and its seven metabolite concentrations were normally distributed. Linear regression was conducted to investigate all pair-wise correlations among tamoxifen metabolite concentrations. The association of genetic activity of CYP2D6 [11] and all other genes with log-transformed tamoxifen metabolite concentrations were tested via univariate linear regression. Suggestive univariate genetic associations $(p<0.1)$ were included in a multivari- 
able backward linear regression model with clinical covariates (age and body mass index (BMI)), which were selected based on their consistent associations with endoxifen and other metabolites [17]. To isolate the effect of genetics on the generation of the metabolite of interest, multivariable linear regression models also included all upstream metabolites. An uncorrected $\alpha=0.05(p<0.05)$ was considered statistically significant for all variables in the multivariable analyses and only statistically significant results are reported. All statistical analyses were performed using IBM SPSS statistical software version 27 (SPSS, Inc., Chicago, IL, USA).

\section{Results}

\subsection{Patient Data and Genetics}

After excluding patients missing concentration or genetic data, patients receiving a daily tamoxifen dose other than $20 \mathrm{mg} /$ day, and non-adherent patients, 187 patients were included in this analysis (Figure 1). Patient data, including demographics, tumor characteristics and treatment regimens, are reported in Table 1 . The median age in this cohort was $48,92.7 \%$ of the patients self-reported as White, and $87 \%$ were pre-menopausal. Geneticdefined metabolic phenotype frequencies for CYP2D6 and all other genotyped genes are reported in Table 2.

\section{Prospective Breast Cancer Biobank $(\mathrm{N}=1455)$}
Inclusion criteria:
$\mathrm{ER}+$ breast cancer
Tamoxifen treatment
Enrolled in side effect study
$(\mathrm{N}=220)$
Serum sample not drawn during
tamoxifen treatment $(n=10)$

\section{Patients eligible for genotyping \\ $(\mathrm{N}=210)$ \\ DNA not available for genotyping $(n=7)$}

\section{Patients available for present study}

$(\mathrm{N}=203)$

Non-adherent to tamoxifen ( $n=15$ )

Not receiving $20 \mathrm{mg} /$ day dose $(n=1)$

\section{Patients included in analysis $(\mathrm{N}=187)$}

Figure 1. Patient flow from original cohort into present study. PROM = Patient Reported Outcomes. Non-adherence was determined by pharmacokinetic tamoxifen cut off $40 \mathrm{nM}$. 
Table 1. Patient Demographics.

\begin{tabular}{ccc}
\hline Clinical Variable & Level & Median (Range) or $\boldsymbol{n}$ (\%) \\
\hline Age & Years & $48.00(24.0-84.0)$ \\
\hline \multirow{2}{*}{ Menopausal Status } & Premenopausal & $162(86.6 \%)$ \\
& Postmenopausal & $25(13.4 \%)$ \\
\hline Body mass index & $\mathrm{kg} / \mathrm{m}^{2}$ & $24.6(17.37-41.14)$ \\
\hline \multirow{2}{*}{ Race } & Asian & $6(3.2 \%)$ \\
& White & $112(59.9 \%)$ \\
Chemotherapy & Other/Unknown & $69(36.9 \%)$ \\
\hline Time on tamoxifen & No & $19(10.2 \%)$ \\
& Yes & $168(89.8 \%)$ \\
\hline \multirow{2}{*}{ Tamoxifen Metabolites } & Months & $12.0(1.0-67.0)$ \\
& Tamoxifen & $296.5(56.17-898.66)$ \\
& NDtam & $584.2450(166.83-1551.44)$ \\
& Z-4OHtam & $5.0170(0.70-22.22)$ \\
& Z-endoxifen & $28.3080(4.45-96.00)$ \\
& Z-4'-endoxifen & $22.9850(6.70-62.97)$ \\
& Z-4'-Ohtam & $7.3110(1.72-23.00)$ \\
& TamNoX & $18.6530(3.60-70.42)$ \\
& NNDDtam & $88.6410(14.90-239.35)$ \\
\hline
\end{tabular}

Menopausal status is age determined (cut off 55 years). Race is determined according to U.S. Census Bureau.

Table 2. Genetically-defined Metabolic Activity Phenotypes.

\begin{tabular}{|c|c|c|c|c|c|c|}
\hline \multirow{2}{*}{ Genes } & \multicolumn{6}{|c|}{ Phenotypes $(n, \%)$} \\
\hline & PM & IM & NM & RM & UM & Missing \\
\hline CYP2D6 & $13(7.0 \%)$ & $65(34.8 \%)$ & 98 (52.4\%) & & $11(5.9 \%)$ & \\
\hline СУРЗА4 & & $22(11.8 \%)$ & $165(88.2 \%)$ & & & \\
\hline СУРЗА5 & 155 (82.9\%) & $28(15.0 \%)$ & $1(0.5 \%)$ & & & $3(1.6 \%)$ \\
\hline CYP2C19 & $5(2.7 \%)$ & $51(27.3 \%)$ & $83(44.4 \%)$ & $42(22.5 \%)$ & $4(2.1 \%)$ & $2(1.1 \%)$ \\
\hline CYP1A2 & & $4(2.1 \%)$ & $21(11.2 \%)$ & & $162(86.6 \%)$ & \\
\hline CYP2C9 & $3(1.6 \%)$ & $63(33.7 \%)$ & $121(64.7 \%)$ & & & \\
\hline CYP2B6 & $11(5.9 \%)$ & $61(32.6 \%)$ & $115(61.5 \%)$ & & & \\
\hline$A B C B 1$ & $30(16.0 \%)$ & $82(43.9 \%)$ & $75(40.1 \%)$ & & & \\
\hline SULT4A1 & $4(2.1 \%)$ & $35(18.7 \%)$ & $148(79.1 \%)$ & & & \\
\hline$A P O E$ & $7(3.7 \%)$ & $7(3.7 \%)$ & $167(89.3 \%)$ & & & $6(3.2 \%)$ \\
\hline COMT & $63(33.7 \%)$ & $88(47.1 \%)$ & $35(18.7 \%)$ & & & $1(0.5 \%)$ \\
\hline DRD2 & $10(5.3 \%)$ & $56(29.9 \%)$ & $120(64.2 \%)$ & & & $1(0.5 \%)$ \\
\hline$F 2$ & & $5(2.7 \%)$ & $182(97.3 \%)$ & & & \\
\hline F5 & & $14(7.5 \%)$ & $173(92.5 \%)$ & & & \\
\hline GLP1R & 39 (20.9\%) & 111 (59.4\%) & $34(18.2 \%)$ & & & $3(1.6 \%)$ \\
\hline MTHFR & $14(7.5 \%)$ & $72(38.5 \%)$ & $101(54.0 \%)$ & & & \\
\hline OPRM1 & $5(2.7 \%)$ & $31(16.6 \%)$ & $151(80.7 \%)$ & & & \\
\hline PNPLA5 & $3(1.6 \%)$ & $40(21.4 \%)$ & $144(77.0 \%)$ & & & \\
\hline SLCO1B1 & $6(3.2 \%)$ & $53(28.3 \%)$ & $127(67.9 \%)$ & & & $1(0.5 \%)$ \\
\hline VKORC1 & $21(11.2 \%)$ & $90(48.1 \%)$ & $76(40.6 \%)$ & & & \\
\hline
\end{tabular}

$\mathrm{PM}=$ Poor Metabolizer, $\mathrm{IM}=$ Intermediate Metabolizer, $\mathrm{NM}=$ Normal Metabolizer, $\mathrm{RM}=$ Rapid Metabolizer, $\mathrm{UM}=$ Ultra-rapid Metabolizer.

\subsection{Concentrations of Tamoxifen Metabolites}

Steady-state concentrations of tamoxifen and all measured metabolites can be found in Table 1. The median steady state concentration of tamoxifen was $296.5 \mathrm{nM}$ and the median concentrations of the active metabolites Z-endoxifen and Z-4OHtam were $28.3 \mathrm{nM}$ and $5.0 \mathrm{nM}$, respectively, in line with other studies $[3,6]$.

Pair-wise correlations between tamoxifen metabolite concentrations are reported in Table 3. Tamoxifen was significantly correlated with all downstream metabolites (all $p<0.01)$. Most metabolites were highly correlated with each other $(p<0.01)$, even those that were not in direct relation, or within, the same metabolic pathway. 
Table 3. Pearson Correlation Between Tamoxifen Metabolites.

\begin{tabular}{|c|c|c|c|c|c|c|c|c|c|}
\hline & & Tamoxifen & NDtam & $\begin{array}{c}\mathrm{Z} 4 \mathrm{OH} \\
\text { tam }\end{array}$ & $\begin{array}{c}\text { Z- } \\
\text { endoxifen }\end{array}$ & $\begin{array}{c}\text { Z-4- } \\
\text { prime- } \\
\text { endoxifen }\end{array}$ & $\begin{array}{c}\text { Z-4- } \\
\text { prime- } \\
\text { OH-tam }\end{array}$ & TamNoX & $\begin{array}{c}\text { NNDD } \\
\text { tam }\end{array}$ \\
\hline \multirow{2}{*}{ Tamoxifen } & Correlation & & 0.735 & 0.648 & 0.442 & 0.504 & 0.758 & 0.75 & 0.601 \\
\hline & $p$-value & & $<0.001$ & $<0.001$ & $<0.001$ & $<0.001$ & $<0.001$ & $<0.001$ & $<0.001$ \\
\hline \multirow{2}{*}{ NDtam } & Correlation & & & 0.312 & 0.136 & 0.835 & 0.741 & 0.548 & 0.689 \\
\hline & $p$-value & & & $<0.001$ & 0.063 & $<0.001$ & $<0.001$ & $<0.001$ & $<0.001$ \\
\hline \multirow{2}{*}{ Z4OHtam } & Correlation & & & & 0.839 & 0.203 & 0.606 & 0.511 & 0.351 \\
\hline & $p$-value & & & & $<0.001$ & 0.005 & $<0.001$ & $<0.001$ & $<0.001$ \\
\hline \multirow{2}{*}{ Z-endoxifen } & Correlation & & & & & -0.029 & 0.346 & 0.385 & 0.385 \\
\hline & $p$-value & & & & & 0.69 & $<0.001$ & $<0.001$ & $<0.001$ \\
\hline \multirow{2}{*}{ Z-4-prime-endoxifen } & Correlation & & & & & & 0.798 & 0.326 & 0.547 \\
\hline & $p$-value & & & & & & $<0.001$ & $<0.001$ & $<0.001$ \\
\hline \multirow{2}{*}{ Z-4-prime-OH-tam } & Correlation & & & & & & & 0.501 & 0.549 \\
\hline & $p$-value & & & & & & & $<0.001$ & $<0.001$ \\
\hline \multirow{2}{*}{ TamNoX } & Correlation & & & & & & & & 0.41 \\
\hline & $p$-value & & & & & & & & $<0.001$ \\
\hline NNDDtam & $\begin{array}{c}\text { Correlation } \\
p \text {-value }\end{array}$ & & & & & & & & \\
\hline
\end{tabular}

\subsection{Genetic Associations with Tamoxifen Metabolites}

Multivariable linear regression was performed including genes with suggestive associations in univariate analysis, upstream metabolites, age and BMI (Table 4). Higher tamoxifen concentrations were associated with lower activity of CYP1A2 and CYP3A4, which, when combined, explained $5.3 \%$ of tamoxifen concentrations. The major pathway responsible for tamoxifen metabolism is demethylation to NDtam. We found that NDtam was inversely associated with CYP2D6 activity, which explained $11 \%$ of the variability in NDtam concentrations. Another route of tamoxifen metabolism is activation to Z-4OHtam. Higher Z-4OHtam concentrations were associated with higher CYP2D6 and CYP2C9 activity, and lower VKORC1 and SLCO1B1 activity, which, together, explained $14.2 \%$ of the $\mathrm{Z}-4 \mathrm{OHtam}$ concentrations.

Table 4. Genetic and Clinical Factors Associated with Tamoxifen Metabolites.

\begin{tabular}{|c|c|c|c|c|c|c|}
\hline $\begin{array}{l}\text { Tamoxifen } \\
\text { Metabolite }\end{array}$ & Variables & $\beta$ Coefficient & Std. Error & $p$-Value & & \\
\hline \multirow{2}{*}{ Tamoxifen } & СУРЗА4 & -0.189 & 0.091 & 0.040 & 0.037 & \multirow{2}{*}{0.05} \\
\hline & CYP1A2 & -0.155 & 0.072 & 0.033 & 0.016 & \\
\hline \multirow{3}{*}{ NDtam } & Tamoxifen & 0.716 & 0.039 & $<0.001$ & 0.540 & \multirow{3}{*}{0.67} \\
\hline & CYP2D6 & -0.174 & 0.022 & $<0.001$ & 0.110 & \\
\hline & BMI & -0.333 & 0.101 & 0.001 & 0.020 & \\
\hline \multirow{6}{*}{ Z-4OHtam } & Tamoxifen & 0.710 & 0.052 & $<0.001$ & 0.412 & \multirow{6}{*}{0.59} \\
\hline & CYP2D6 & 0.208 & 0.030 & $<0.001$ & 0.111 & \\
\hline & CYP2C9 & 0.171 & 0.041 & $<0.001$ & 0.015 & \\
\hline & VKORC1 & -0.068 & 0.032 & 0.035 & 0.015 & \\
\hline & SLCO1B1 & -0.085 & 0.039 & 0.032 & 0.001 & \\
\hline & BMI & -0.551 & 0.135 & $<0.001$ & 0.036 & \\
\hline \multirow{4}{*}{ Z-Endoxifen } & Tamoxifen & -0.349 & 0.110 & 0.002 & 0.003 & \multirow{4}{*}{0.79} \\
\hline & NDtam & 0.310 & 0.101 & 0.002 & 0.017 & \\
\hline & 4OHtam & 1.038 & 0.072 & $<0.001$ & 0.703 & \\
\hline & CYP2D6 & 0.361 & 0.037 & $<0.001$ & 0.070 & \\
\hline \multirow{4}{*}{ Z-4'-Endoxifen } & NDtam & 0.989 & 0.067 & $<0.001$ & 0.699 & \multirow{4}{*}{0.75} \\
\hline & Tamoxifen & -0.299 & 0.060 & $<0.001$ & 0.029 & \\
\hline & CYP2D6 & -0.046 & 0.023 & 0.048 & 0.010 & \\
\hline & BMI & -0.279 & 0.096 & 0.004 & 0.012 & \\
\hline \multirow{2}{*}{ Z-4'-OH-tam } & Tamoxifen & 0.680 & 0.041 & $<0.001$ & 0.576 & \multirow{2}{*}{0.61} \\
\hline & BMI & -0.383 & 0.107 & $<0.001$ & 0.034 & \\
\hline
\end{tabular}


Table 4. Cont.

\begin{tabular}{|c|c|c|c|c|c|c|}
\hline $\begin{array}{l}\text { Tamoxifen } \\
\text { Metabolite }\end{array}$ & Variables & $\beta$ Coefficient & Std. Error & $p$-Value & & \\
\hline \multirow{3}{*}{ TamNoX } & Tamoxifen & 1.026 & 0.049 & $<0.001$ & 0.563 & \multirow{3}{*}{0.5} \\
\hline & SLCO1B1 & -0.162 & 0.037 & $<0.001$ & 0.018 & \\
\hline & CYP3A4 & 0.191 & 0.061 & 0.002 & 0.012 & \\
\hline \multirow{4}{*}{ NNDDtam } & NDtam & 0.972 & 0.054 & $<0.001$ & 0.490 & \multirow{4}{*}{0.62} \\
\hline & CYP2D6 & 0.183 & 0.029 & $<0.001$ & 0.083 & \\
\hline & СYР3A4 & 0.243 & 0.061 & $<0.001$ & 0.025 & \\
\hline & CYP2C19 & -0.075 & 0.024 & 0.002 & 0.025 & \\
\hline
\end{tabular}

Endoxifen is formed through two pathways, primarily by hydroxylation of NDtam, or via the minor route of demethylation of $\mathrm{Z}-4 \mathrm{OH}$ tam. In the univariate linear regression, CYP1A2, CYP2D6, F5, SLCO1B1 and VKORC1, all had suggestive associations $(p<0.1$, data not shown), but after backward linear regression, endoxifen concentrations were only associated with tamoxifen and the two intermediate metabolites, and increased CYP2D6 activity (combined $\mathrm{r}^{2}=0.79$ ).

Next, the isomeride forms of Z-endoxifen and Z-4OHtam were studied. After backward linear regression, $Z-4^{\prime}$-endoxifen concentrations were inversely associated with CYP2D6 activity, whereas no genes were significantly associated with Z-4'-OHtam concentrations. The other tamoxifen metabolites, TamNoX and NNDDtam, were also measured. Higher TamNoX concentrations were found in patients with increased CYP3A4 or decreased SLCO1B1 activity. Higher NNDDTam concentrations were associated with increased CYP3A4 and CYP2D6 activity, and decreased CYP2C19 activity.

\section{Discussion}

There is evidence that the pharmacological activity of tamoxifen is determined by its conversion to endoxifen $[5,6,18,19]$, though this has not been consistently demonstrated $[2,20]$. These inconsistent findings may be partially due to the contribution of other active metabolites, such as $4 \mathrm{OHtam}[11,21-23]$, which has also been reported to affect treatment efficacy $[3,4]$, and perhaps other, less active metabolites such as Z-4'-endoxifen and Z-4'-OHtam [7]. As is described in detail in our recent review [12], substantial work has been carried out to identify the genetic variables associated with endoxifen concentrations, but much less work has been conducted to identify the genetic variants that affect non-endoxifen metabolites. To investigate the genes associated with the tamoxifen metabolism pathway, which could facilitate further study of the efficacy of individualized tamoxifen treatment, we conducted an assessment of the effect of genetic variation in 20 pharmacogenes on steady-state concentrations of tamoxifen and its metabolites. This approach allowed us to generate a more complete understanding of the genes responsible for the metabolic conversion of tamoxifen to its downstream metabolites (Figure 2).

Tamoxifen concentrations were higher in patients with lower activity of CYP3A4 and CYP1A2. CYP3A4 is the primary enzyme responsible for the conversion of tamoxifen to its major metabolite, NDtam $[11,25]$. Our results agree with prior evidence that patients with reduced CYP3A4 activity have higher steady-state tamoxifen concentrations, perhaps due to the reduced first-pass metabolism and increased bioavailability [26-28]. CYP1A2 is responsible for demethylation of tamoxifen to NDtam [11], which could explain our finding, since lower CYP1A2 activity leads to less conversion to NDtam. The conversion of tamoxifen to NDtam includes other enzymes, such as CYP2D6 [11], which likely explains our finding that this gene was associated with the NDtam concentration. However, associations with other genes that have previously been reported to be associated with the formation of NDtam, including CYP3A5, CYP2C9/19 [11,25,29], were not identified in our analysis. This may be due to differences between patient cohorts, including race, or the limited size of our cohort to detect genes with smaller effects. 


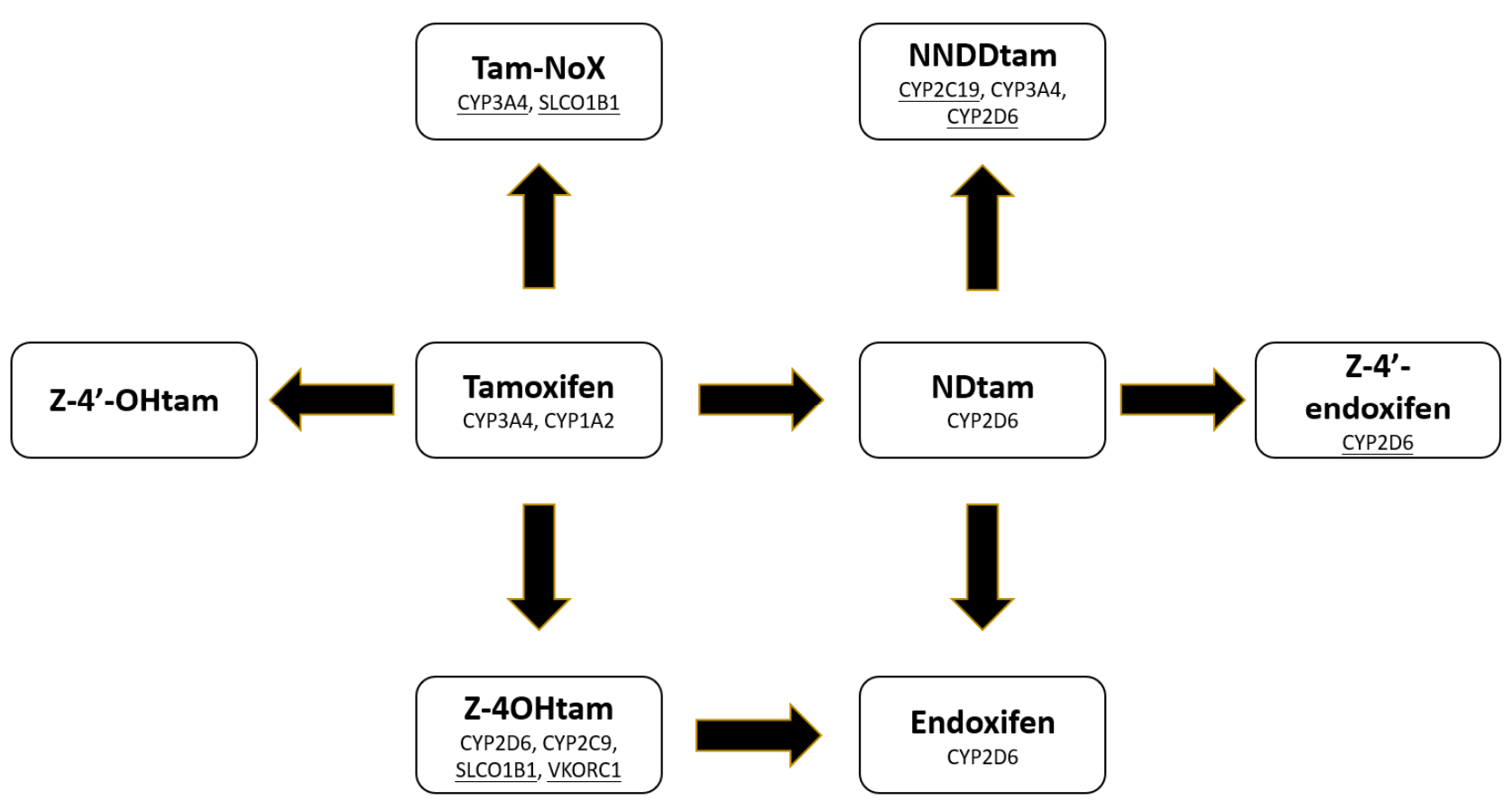

Figure 2. Tamoxifen metabolism pathway and genes associated with each step based on the results of this analysis. Genes associated with each metabolite are indicated within the figure. Normal text indicates our data confirm prior evidence of the gene's contribution to this metabolic conversion [11,24], whereas underlined text indicates a finding that has not been previously described to our knowledge.

An alternative pathway for tamoxifen metabolism is hydroxylation to Z-4OHtam, which is primarily catalyzed by CYP2D6 [23,30] with a minor role for CYP2C9 [11]. Our findings that higher CYP2D6 and CYP2C9 activity are associated with increased $4 \mathrm{OH}$ tam concentrations are consistent in direction and magnitude with previous studies $[5,11,23]$. We also found weak associations for SLCO1B1 and VKORC1. SLCO1B1 encodes the organic anion-transporter polypeptide 1B1 (OATP1B1), an influx transporter on the sinusoidal membrane of hepatocytes [31]. OATP1B1 transports tamoxifen and endoxifen [32], but our results found that decreased SLCO1B1 activity is associated with higher 4-OHtam levels. This may indicate that 4-OHtam is also transported by OATP1B1, though this has not been studied to our knowledge. VKORC1 encodes the catalytic subunit of the vitamin K epoxide reductase complex, which facilitates the activation of vitamin $\mathrm{K}$. The association for VKORC1 is difficult to explain mechanistically and may be a false positive, due to the many association tests conducted without proper statistical correction. The final step in the metabolic pathway from tamoxifen to endoxifen is the hydroxylation of NDtam to endoxifen [29,30]. Our results agree with the extensive prior literature [12] that patients with greater CYP2D6 activity have higher steady-state endoxifen concentrations. Several studies have reported an association between CYP2C9/19 and endoxifen levels [12]; however, this association was not identified in our analyses. CYP2C9/19 is responsible for generation of $\mathrm{Z}-4 \mathrm{OHtam}$ and $\mathrm{CYP} 2 \mathrm{C} 19$ is involved in the demethylation of Z-4OHtam to endoxifen. Since our regression analysis included Z-4OHtam levels the effect of CYP2C9/19 was likely removed. The adjustment of upstream metabolites could eliminate the upstream effect and isolate the genes that actually contribute to the metabolic conversion of interest.

We also investigated associations of pharmacogenes with the 4-prime Z-isomers of endoxifen (i.e., Z-4'-Endoxifen) and $4 \mathrm{OHtam}$ (i.e., Z-4'-OH-tam), which have a lower binding affinity to the estrogen receptor [7]. The genes responsible for the metabolism of NDtam to Z-4'-Endoxifen are unknown. Our analysis indicates an inverse relationship between CYP2D6 activity and Z-4'-Endoxifen; however, this is likely due to the role of CYP2D6 in generating the Z-isomers, thereby leaving more substrate (NDtam) available 
for Z-4'-endoxifen generation in patients with low CYP2D6 activity. Our analysis did not identify any genes associated with Z-4'-OHtam after backward linear regression.

Finally, our study analyzed associations for two secondary metabolites with low or unknown anti-estrogenic effects: NNDDtam and TamNoX. CYP3A4 and CYP3A5 are believed to be responsible for the demethylation of NDtam to NNDDtam [11,24], which is consistent with our findings that CYP3A4 is associated with NNDDtam concentrations. There may have been insufficient genetic heterogeneity in CYP3A5 to detect this association, or CYP3A5 may play a very minor role in this metabolic pathway. Interestingly, we also found stronger contributions from CYP2D6 and CYP2C19, which are involved in the formation of the upstream metabolite NDtam $[11,25,29]$ but not known to be involved in its downstream metabolism to NNDDtam. The enzymes previously thought to be responsible for the formation of TamNoX, FMO1 and FMO3 [24], were not included in our analysis. Our results indicated that CYP3A4 and SLCO1B1 were associated with TamNoX concentrations, but both explained a minimal portion of the variability after accounting for tamoxifen.

The effect of genetic variation on concentrations of tamoxifen and its metabolites may be useful to patients if the relationship between endoxifen, or some combination of its active metabolites, with tamoxifen efficacy is validated. The high correlation among the tamoxifen metabolites will make it challenging to identify the active metabolite or optimal combination, but that does not mean that a predictive biomarker could not be validated. Upon validation, genetic and clinical variables could be used to determine the optimal starting dose of tamoxifen [12], perhaps followed by a therapeutic drug monitoring approach to achieve the active metabolite concentration target [33], which could aid in improving the outcome of tamoxifen treatment such as the ten-year survival and recurrence rate. A recent study used panel genotyping to generate a population pharmacokinetic (PopPK) model that indicated CYP2D6 IM and PM patients would require increases to $40 \mathrm{mg} /$ day and $80 \mathrm{mg} /$ day to achieve endoxifen concentrations similar to those seen in CYP2D6 NM on $20 \mathrm{mg} /$ day [34], which is consistent with the results of CYP2D6-guided tamoxifen dose escalation studies [35].

The strengths of our study included accurate quantification of tamoxifen and several of its major metabolites using a highly selective and sensitive UPLC-MS/MS assay [3,14]. Thus, the genotypic variants are only surrogate markers of the plasma concentration of the tamoxifen metabolites. These comprehensive metabolite data were combined with comprehensive genotyping for CYP2D6, including common variants and copy number variation, and 20 additional pharmacogenes. We also employed a novel approach of adjusting for upstream metabolites to isolate the effect of genetics on the metabolite of interest. However, this study had several limitations that should be considered. Some non-CYP genes previously reported to be associated with tamoxifen metabolism were not included in the pharmacogene panels used for genotyping, and therefore were not included in our analysis, including SULT1A1 [36] and UGTs including UGT1A1, UGT2B7 and UGT2B15 [37-39]. Additionally, our cohort of $\sim 200$ patients from Norway lacks genetic heterogeneity for some genes and variants (e.g., CYP3A5*3) found primarily in non-Caucasians. Further research should be done in larger and more diverse cohorts to generate an algorithm that can integrate clinical and genetic data to precisely predict a patient's steady-state concentrations of active tamoxifen metabolites.

\section{Conclusions}

This pharmacogenetic analysis confirmed that genetic variation in CYP2D6 is the primary determinant of tamoxifen metabolism, with a secondary contribution from several other previously known genes including CYP3A4 and CYP2C9. SLCO1B1, which encodes the OATP1B1 transporter, was identified as a novel gene that is associated with active tamoxifen metabolite concentrations. Upon validation that tamoxifen metabolites determine treatment efficacy, individualized treatment should be developed that integrates all relevant genetic and clinical information to select the appropriate starting doses. Fu- 
ture research is needed in larger and more diverse cohorts with more genetic and clinical information to develop a more precise algorithm for tamoxifen metabolism that can be used to improve treatment outcomes in patients with $\mathrm{HR}+$ breast cancer in the absence of metabolite measurement.

Supplementary Materials: The following are available online at https: / www.mdpi.com/article / 10.3390/jpm11060507/s1, Table S1: Allelic Activity Designations. CYP2D6 adapted from CPIC guideline. Table S2: Phenotype Activity Designations. CYP2D6 adapted from CPIC guideline [40].

Author Contributions: Conceptualization, D.L.H., L.A.M. and T.H.; data curation, T.H. and L.A.M., T.H.L., H.S., G.M.; data clean, L.A.M., Y.C., and D.L.H.; data analysis, Y.C., T.H. and D.L.H.; writingoriginal draft preparation, Y.C. and T.H.; writing-review and editing, D.L.H., T.H., L.A.M., H.S., G.M., F.M.E., T.H.L. and Y.C.; visualization, Y.C., T.H., D.L.H.; supervision, D.L.H.; project administration, D.L.H.; funding acquisition, H.S., G.M., D.L.H. All authors have read and agreed to the published version of the manuscript.

Funding: This work was funded by the Western Norwegian Health Authority Grant number: F-10450 and the U.S.- Norway Fulbright Foundation.

Institutional Review Board Statement: Not Applicable.

Informed Consent Statement: Not Applicable.

Data Availability Statement: The data presented in this study may be available on reasonable request from the corresponding author. The data are not publicly available due to legal/privacy restrictions regarding patient data.

Acknowledgments: We would like to thank all the patients that currently are, or have been, participating in the Prospective Breast Cancer Biobank (PBCB) project.

Conflicts of Interest: The authors declare no conflict of interest.

\section{References}

1. Early Breast Cancer Trialists' Collaborative Group (EBCTCG). Effects of chemotherapy and hormonal therapy for early breast cancer on recurrence and 15-year survival: An overview of the randomised trials. Lancet 2005, 365, 1687-1717. [CrossRef]

2. Johnson, M.D.; Zuo, H.; Lee, K.-H.; Trebley, J.P.; Rae, J.M.; Weatherman, R.V.; Desta, Z.; Flockhart, D.A.; Skaar, T.C. Pharmacological Characterization of 4-hydroxy-N-desmethyl Tamoxifen, a Novel Active Metabolite of Tamoxifen. Breast Cancer Res. Treat. 2004, 85, 151-159. [CrossRef] [PubMed]

3. Helland, T.; Henne, N.; Bifulco, E.; Naume, B.; Borgen, E.; Kristensen, V.N.; Kvaløy, J.T.; Lash, T.L.; Alnæs, G.I.G.; van Schaik, R.H.; et al. Serum concentrations of active tamoxifen metabolites predict long-term survival in adjuvantly treated breast cancer patients. Breast Cancer Res. 2017, 19, 125. [CrossRef]

4. Helland, T.; Naume, B.; Hustad, S.; Bifulco, E.; Kvaløy, J.T.; Saetersdal, A.B.; Synnestvedt, M.; Lende, T.H.; Gilje, B.; Mjaaland, I.; et al. Low Z-4OHtam concentrations are associated with adverse clinical outcome among early stage premenopausal breast cancer patients treated with adjuvant tamoxifen. Mol. Oncol. 2021, 15, 957-967. [CrossRef] [PubMed]

5. Saladores, P.; Mürdter, T.; Eccles, D.; Chowbay, B.; Zgheib, N.K.; Winter, S.; Ganchev, B.; Eccles, B.; Gerty, S.; Tfayli, A.; et al. Tamoxifen metabolism predicts drug concentrations and outcome in premenopausal patients with early breast cancer. Pharm. J. 2015, 15, 84-94. [CrossRef]

6. Madlensky, L.; Natarajan, L.; Tchu, S.; Pu, M.; Mortimer, J.; Flatt, S.W.; Nikoloff, D.M.; Hillman, G.; Fontecha, M.R.; Lawrence, H.J.; et al. Tamoxifen metabolite concentrations, CYP2D6 genotype, and breast cancer outcomes. Clin. Pharmacol. Ther. 2011, 89, 718-725. [CrossRef] [PubMed]

7. Barginear, M.F.; Jaremko, M.; Peter, I.; Yu, C.; Kasai, Y.; Kemeny, M.; Raptis, G.; Desnick, R.J. Increasing tamoxifen dose in breast cancer patients based on CYP2D6 genotypes and endoxifen levels: Effect on active metabolite isomers and the antiestrogenic activity score. Clin. Pharmacol. Ther. 2011, 90, 605-611. [CrossRef]

8. Jager, N.G.L.; Rosing, H.; Linn, S.C.; Schellens, J.H.M.; Beijnen, J.H. Importance of highly selective LC-MS/MS analysis for the accurate quantification of tamoxifen and its metabolites: Focus on endoxifen and 4-hydroxytamoxifen. Breast Cancer Res. Treat. 2012, 133, 793-798. [CrossRef]

9. de Vries Schultink, A.H.M.; Alexi, X.; van Werkhoven, E.; Madlensky, L.; Natarajan, L.; Flatt, S.W.; Zwart, W.; Linn, S.C.; Parker, B.A.; Wu, A.H.B.; et al. An Antiestrogenic Activity Score for tamoxifen and its metabolites is associated with breast cancer outcome. Breast Cancer Res. Treat. 2017, 161, 567-574. [CrossRef]

10. Gaedigk, A.; Ingelman-Sundberg, M.; Miller, N.A.; Leeder, J.S.; Whirl-Carrillo, M.; Klein, T.E.; PharmVar Steering Committee. The Pharmacogene Variation (PharmVar) Consortium: Incorporation of the Human Cytochrome P450 (CYP) Allele Nomenclature Database. Clin. Pharmacol. Ther. 2018, 103, 399-401. [CrossRef] 
11. Mürdter, T.E.; Schroth, W.; Bacchus-Gerybadze, L.; Winter, S.; Heinkele, G.; Simon, W.; Fasching, P.A.; Fehm, T.; German Tamoxifen and AI Clinicians Group; Eichelbaum, M.; et al. Activity levels of tamoxifen metabolites at the estrogen receptor and the impact of genetic polymorphisms of phase I and II enzymes on their concentration levels in plasma. Clin. Pharmacol. Ther. 2011, 89, 708-717. [CrossRef]

12. Helland, T.; Alsomairy, S.; Lin, C.; Søiland, H.; Mellgren, G.; Hertz, D.L. Generating a Precision Endoxifen Prediction Algorithm to Advance Personalized Tamoxifen Treatment in Patients with Breast Cancer. J. Pers. Med. 2021, 11, 201. [CrossRef] [PubMed]

13. Lunde, S.; Helland, T.; Jonassen, J.; Haugstøyl, M.; Austdal, M.; Lode, K.; Hagen, K.; Gripsrud, B.; Lind, R.; Gjerde, J.; et al. A Prospective, Longitudinal, Breast Cancer Biobank (PBCB) in Western Norway. European Biobank Week (EBW). 2018. [CrossRef]

14. Helland, T.; Hagen, K.B.; Haugstøyl, M.E.; Kvaløy, J.T.; Lunde, S.; Lode, K.; Lind, R.A.; Gripsrud, B.H.; Jonsdottir, K.; Gjerde, J.; et al. Drug monitoring of tamoxifen metabolites predicts vaginal dryness and verifies a low discontinuation rate from the Norwegian Prescription Database. Breast Cancer Res. Treat. 2019, 177, 185-195. [CrossRef] [PubMed]

15. Marcath, L.A.; Kidwell, K.M.; Robinson, A.C.; Vangipuram, K.; Burness, M.L.; Griggs, J.J.; Poznak, C.V.; Schott, A.F.; Hayes, D.F.; Henry, N.L.; et al. Patients carrying CYP2C8*3 have shorter systemic paclitaxel exposure. Pharmacogenomics 2019, 20, 95-104. [CrossRef]

16. Caudle, K.E.; Sangkuhl, K.; Whirl-Carrillo, M.; Swen, J.J.; Haidar, C.E.; Klein, T.E.; Gammal, R.S.; Relling, M.V.; Scott, S.A.; Hertz, D.L.; et al. Standardizing CYP2D6 Genotype to Phenotype Translation: Consensus Recommendations from the Clinical Pharmacogenetics Implementation Consortium and Dutch Pharmacogenetics Working Group. Clin. Transl. Sci. 2020, 13, 116-124. [CrossRef]

17. Sanchez-Spitman, A.B.; Swen, J.J.; Dezentje, V.O.; Moes, D.J.A.R.; Gelderblom, H.; Guchelaar, H.J. Clinical pharmacokinetics and pharmacogenetics of tamoxifen and endoxifen. Expert Rev. Clin. Pharmacol. 2019, 12, 523-536. [CrossRef]

18. Yang, G.; Nowsheen, S.; Aziz, K.; Georgakilas, A.G. Toxicity and adverse effects of Tamoxifen and other anti-estrogen drugs. Pharmacol. Ther. 2013, 139, 392-404. [CrossRef]

19. Neven, P.; Jongen, L.; Lintermans, A.; Van Asten, K.; Blomme, C.; Lambrechts, D.; Poppe, A.; Wildiers, H.; Dieudonné, A.-S.; Brouckaert, O.; et al. Tamoxifen Metabolism and Efficacy in Breast Cancer: A Prospective Multicenter Trial. Clin. Cancer Res. Off. J. Am. Assoc. Cancer Res. 2018, 24, 2312-2318. [CrossRef] [PubMed]

20. Wakeling, A.E.; Slater, S.R. Estrogen-receptor binding and biologic activity of tamoxifen and its metabolites. Cancer Treat. Rep. 1980, 64, 741-744.

21. Katzenellenbogen, B.S.; Norman, M.J.; Eckert, R.L.; Peltz, S.W.; Mangel, W.F. Bioactivities, estrogen receptor interactions, and plasminogen activator-inducing activities of tamoxifen and hydroxy-tamoxifen isomers in MCF-7 human breast cancer cells. Cancer Res. 1984, 44, 112-119. [PubMed]

22. Ahmad, I. Tamoxifen a pioneering drug: An update on the therapeutic potential of tamoxifen derivatives. Eur. J. Med. Chem. 2018, 143, 515-531.

23. Brauch, H.; Jordan, V.C. Targeting of tamoxifen to enhance antitumour action for the treatment and prevention of breast cancer: The 'personalised' approach? Eur. J. Cancer 2009, 45, 2274-2283. [CrossRef]

24. Brauch, H.; Mürdter, T.E.; Eichelbaum, M.; Schwab, M. Pharmacogenomics of tamoxifen therapy. Clin. Chem. 2009, 55, 1770-1782. [CrossRef]

25. Klein, D.J.; Thorn, C.F.; Desta, Z.; Flockhart, D.A.; Altman, R.B.; Klein, T.E. PharmGKB summary: Tamoxifen pathway, pharmacokinetics. Pharm. Genom. 2013, 23, 643-647. [CrossRef]

26. Teft, W.A.; Gong, I.Y.; Dingle, B.; Potvin, K.; Younus, J.; Vandenberg, T.A.; Brackstone, M.; Perera, F.E.; Choi, Y.-H.; Zou, G.; et al. CYP3A4 and seasonal variation in vitamin D status in addition to CYP2D6 contribute to therapeutic endoxifen level during tamoxifen therapy. Breast Cancer Res. Treat. 2013, 139, 95-105. [CrossRef]

27. Antunes, M.V.; de Oliveira, V.; Raymundo, S.; Staudt, D.E.; Gössling, G.; Biazús, J.V.; Cavalheiro, J.A.; Rosa, D.D.; Mathy, G.; Wallemacq, P.; et al. CYP3A4*22 is related to increased plasma levels of 4-hydroxytamoxifen and partially compensates for reduced CYP2D6 activation of tamoxifen. Pharmacogenomics 2015, 16, 601-617. [CrossRef] [PubMed]

28. Antunes, M.V.; Timm, T.A.d.F.; de Oliveira, V.; Staudt, D.E.; Raymundo, S.; Gössling, G.; Biazús, J.V.; Cavalheiro, J.A.; Rosa, D.D.; Wallemacq, P.; et al. Influence of CYP2D6 and CYP3A4 Phenotypes, Drug Interactions, and Vitamin D Status on Tamoxifen Biotransformation. Ther. Drug Monit. 2015, 37, 733-744. [CrossRef]

29. Desta, Z.; Ward, B.A.; Soukhova, N.V.; Flockhart, D.A. Comprehensive evaluation of tamoxifen sequential biotransformation by the human cytochrome P450 system in vitro: Prominent roles for CYP3A and CYP2D6. J. Pharmacol. Exp. Ther. 2004, 310, 1062-1075. [CrossRef] [PubMed]

30. Cronin-Fenton, D.P.; Damkier, P. Tamoxifen and CYP2D6: A Controversy in Pharmacogenetics. Adv. Pharmacol. $2018,83,65-91$.

31. Kalliokoski, A.; Niemi, M. Impact of OATP transporters on pharmacokinetics. Br. J. Pharmacol. 2009, 158, 693-705. [CrossRef]

32. Gao, C.-M.; Pu, Z.; He, C.; Liang, D.; Jia, Y.; Yuan, X.; Wang, G.; Xie, H. Effect of OATP1B1 genetic polymorphism on the uptake of tamoxifen and its metabolite, endoxifen. Oncol. Rep. 2017, 38, 1124-1132. [CrossRef] [PubMed]

33. Fox, P.; Balleine, R.L.; Lee, C.; Gao, B.; Balakrishnar, B.; Menzies, A.M.; Yeap, S.H.; Ali, S.S.; Gebski, V.; Provan, P.; et al. Dose Escalation of Tamoxifen in Patients with Low Endoxifen Level: Evidence for Therapeutic Drug Monitoring-The TADE Study. Clin. Cancer Res. 2016, 22, 3164-3171. [CrossRef] [PubMed] 
34. Puszkiel, A.; Arellano, C.; Vachoux, C.; Evrard, A.; Le Morvan, V.; Boyer, J.-C.; Robert, J.; Delmas, C.; Dalenc, F.; Debled, M.; et al. Model-Based Quantification of Impact of Genetic Polymorphisms and Co-Medications on Pharmacokinetics of Tamoxifen and Six Metabolites in Breast Cancer. Clin. Pharmacol. Ther. 2021, 109, 1244-1255. [CrossRef] [PubMed]

35. Hertz, D.L.; Deal, A.; Ibrahim, J.G.; Walko, C.M.; Weck, K.E.; Anderson, S.; Magrinat, G.; Olajide, O.; Moore, S.; Raab, R.; et al. Tamoxifen Dose Escalation in Patients With Diminished CYP2D6 Activity Normalizes Endoxifen Concentrations without Increasing Toxicity. Oncologist 2016, 21, 795-803. [CrossRef] [PubMed]

36. Sanchez-Spitman, A.B.; Dezentjé, V.O.; Swen, J.J.; Moes, D.J.a.R.; Gelderblom, H.; Guchelaar, H.-J. Genetic polymorphisms of 3'-untranslated region of SULT1A1 and their impact on tamoxifen metabolism and efficacy. Breast Cancer Res. Treat. 2018, 172, 401-411. [CrossRef]

37. Blevins-Primeau, A.S.; Sun, D.; Chen, G.; Sharma, A.K.; Gallagher, C.J.; Amin, S.; Lazarus, P. Functional significance of UDPglucuronosyltransferase variants in the metabolism of active tamoxifen metabolites. Cancer Res. 2009, 69, 1892-1900. [CrossRef]

38. Lazarus, P.; Sun, D. Potential role of UGT pharmacogenetics in cancer treatment and prevention: Focus on tamoxifen and aromatase inhibitors. Drug Metab. Rev. 2010, 42, 182-194. [CrossRef]

39. Sutiman, N.; Lim, J.S.L.; Muerdter, T.E.; Singh, O.; Cheung, Y.B.; Ng, R.C.H.; Yap, Y.S.; Wong, N.S.; Ang, P.C.S.; Dent, R.; et al. Pharmacogenetics of UGT1A4, UGT2B7 and UGT2B15 and Their Influence on Tamoxifen Disposition in Asian Breast Cancer Patients. Clin. Pharm. 2016, 55, 1239-1250. [CrossRef]

40. Goetz, M.P.; Sangkuhl, K.; Guchelaar, H.-J.; Schwab, M.; Province, M.; Whirl-Carrillo, M.; Symmans, W.F.; McLeod, H.L.; Ratain, M.J.; Zembutsu, H.; et al. Clinical Pharmacogenetics Implementation Consortium (CPIC) Guideline for CYP2D6 and Tamoxifen Therapy. Clin. Pharmacol. Ther. 2018, 103, 770-777. [CrossRef] 